\title{
ПАРАМЕТРЫ ВЗАИМНЫХ ВЛИЯНИЙ LAN-кабелей в широком
}

\section{диапазоне частот}

В.Андреев, д.т.н., президент Поволжского государственного университета телекоммуникаций и информатики;

В.Баннов, к.т.н., технический директор AO "Самарская Кабельная Компания";

В.Ключников, К.Т.н., генеральный директор АO "Самарская Кабельная Компания";

Б.Попов, К.т.н., профессор Поволжского государственного университета телекоммуникаций и информатики;

в.Попов, к.т.н., профессор Поволжского государственного университета телекоммуникаций и информатики / inkat@inbox.ru

УДК 621.396.2, DOI: 10.22184/2070-8963.2020.90.5.14.19

Приводятся результаты экспериментальных исследований параметров взаимного влияния четырехи многопарных LAN-кабелей категории 5е в диапазоне частот до 100 МГц. Показано, что в четырехпарных кабелях нормы на переходное затухание на ближнем и на дальнем концах выполняются с запасом в 5-10 дБ. В многопарных кабелях нормы на эти параметры хотя и выполняются, но с небольшим запасом. Установлено, что у многопарных кабелей на указанные параметры по причине механических воздействий влияют особенности технологии скрутки сердечника из четырехпарных пучков на крутильной машине. Даны рекомендации по минимизации механических воздействий на кабельный сердечник в процессе производства.

\section{ВВЕДЕНИЕ}

Сегодня в России в отрасли инфокоммуникаций наибольшие капитальные вложения направляются на развитие широкополосного доступа (ШПД). Весьма востребованы также структурирован ные кабельные системы (CKC), которые являются неотъемлемой частью любого современного общественного объекта, в первую очередь офисных зданий. В этих сферах широкое применение находит специальная группа медножильных симметричных кабелей - LAN-кабели (кабели "витая пара") - емкостью преимущественно до 25 пар.
В настоящее время наибольшим спросом пользуются четырехпарные кабели без экрана типа U/UTP $4 \times 2 \times 0,52$ категории 5е, предназначен ные для передачи информации на скоростях до 1 Гбит/с с полосой пропускания до 100 МГц. В СКС длина таких кабелей не превышает 100 м, а на сетях ШПД, построенных на основе наиболее экономичной артитектуры FTTB, обычно ограничивается 200-250 м.

Многопарные кабели емкостью $25 \times 2$ пар скручиваются из четырехпарных пучков. Основные потребители этой продукции - операторы связи, 
использующие ее для строительства и ремонта сетей ШПД.

Для стандартизации LAN-кабелей специалистами ВНИИКП разработан ГОСТ Р 54429-2011 [1], в основу которого положен международный стандарт ISO/IEC 11801 [2]. В упомянутом ГОСТ Р приведены нормы на электрические характеристики этих кабелей.

При изготовлении симметричных кабелей в целом и LAN-кабелей в частности всегда принимаются меры для того, чтобы обеспечить уменьшение взаимных влияний между кабельными цепями, так как именно эти влияния являются определяющим фактором при решении задачи достижения высокой скорости передачи по линии связи. Основное внимание при этом уделяется обеспечению симметрии конструкции цепей как в отношении геометрического расположения жил, так и однородности диэлектрических свойств изоляции [3]. Существенное влияние на электрические характеристики оказывает также технология скрутки кабельных цепей. Оба эти фактора и определяют величину электромагнитных влияний между цепями. Получается, что высокие и стабильные электрические характеристики LAN-кабелей можно обеспечить только применением современного технологического оборудования, оснащенного системами автоматического регулирования параметров качества кабеля, а также использованием материалов надежных отечественных поставщиков.

В 2019 году в АО "Самарская кабельная компания" (СКK) начато серийное производство LANкабелей. Для выпуска этой продукции приобретено современное импортное высокоскоростное специализированное оборудование скрутки пар, обеспечивающее высокое качество четырехпарного кабеля категории 5е типа U/UTP 4×2×0,52. Практика производства симметричных кабелей связи показывает, что параметры взаимных влияний между цепями весьма чувствительны к неизбежно возникающим геометрическим неоднородностям. В этой связи возникает практический интерес $\mathrm{K}$ исследованию параметров взаимного влияния LAN-кабелей.

\section{РЕЗУЛЬТАТЫ ИЗМЕРЕНИЯ ПАРАМЕТРОВ ВЗАИМНОГО ВЛИЯНИЯ LAN-КАБЕЛЕЙ}

В классической теории взаимных влияний $[4,5]$ нормирование переходного затухания на ближнем конце и защищенности на дальнем конце кабеля выполнено на величину строительной длины и усилительного (регенерационного) участка. B LANкабелях принято нормировать практически все характеристики, в том числе и характеристики взаимного влияния, на длину 100 м. Поскольку в действующем ГОСТ Р 54529-2011 нормирование и обозначение электрических характеристик LAN-кабелей аналогичны стандарту ISO/IEC 11801, в настоящей статье все обозначения соответствуют современной международной практике. Нормирование параметров взаимного влияния LAN-кабелей выполняется как для отдельных комбинаций цепи, так и для суммарного значения помехи при учете влияния всех цепей кабеля на каждую отдельную цепь. В реальных условиях в кабеле рабочими являются все пары, поэтому наиболее целесообразно измерять суммарное значение помех.

Рассмотрим основные параметры взаимного влияния четырехпарного кабеля типа

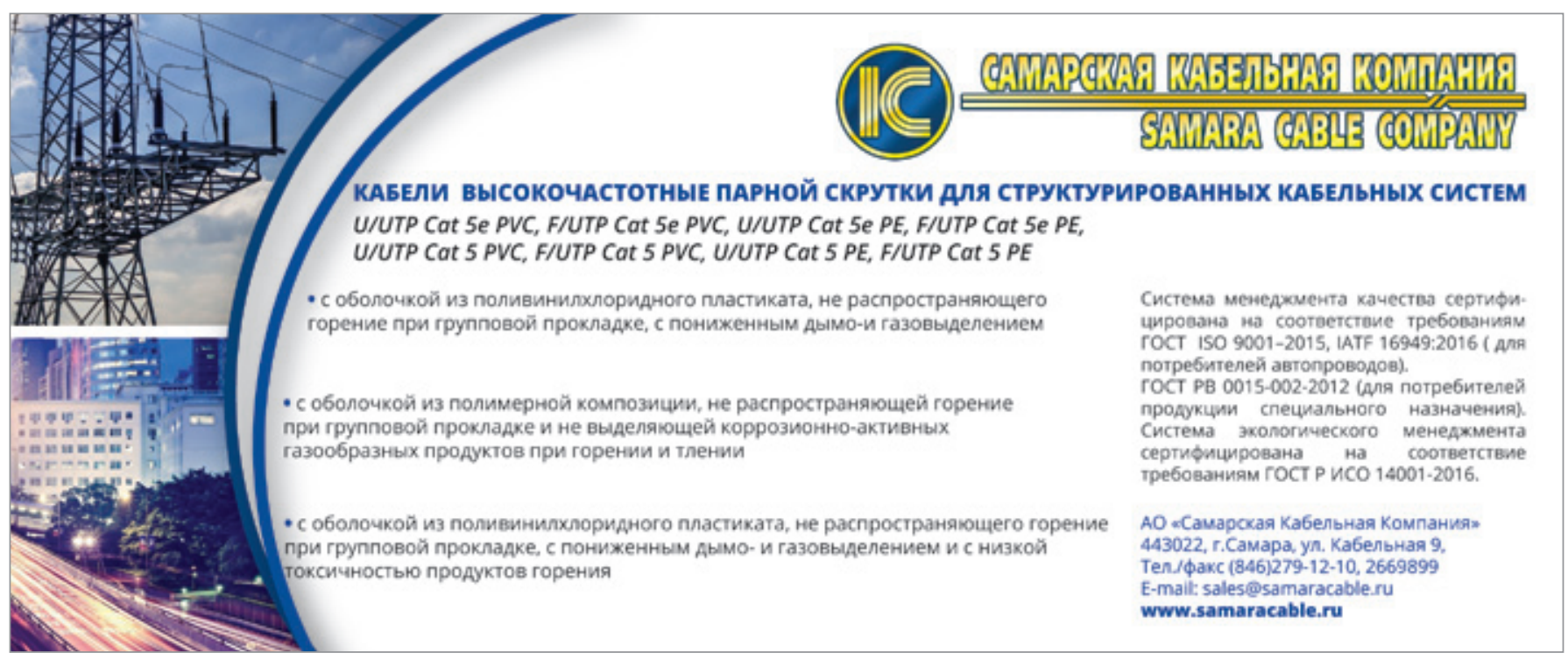


Таблица 1. Низкочастотные характеристики кабеля

\begin{tabular}{|c|c|c|c|c|c|c|c|}
\hline \multicolumn{2}{|c|}{ R, Ом/км } & \multicolumn{2}{|c|}{$\Delta \mathrm{R}, \%$} & \multicolumn{2}{|c|}{ С, нФ/км } & \multicolumn{2}{|c|}{ Е, пФ/км } \\
\hline $\mathrm{R}_{\mathrm{cp}}$ & $\sigma$ & $\Delta \mathrm{R}_{\mathrm{cp}}$ & $\sigma$ & $C_{c p}$ & $\sigma$ & $E_{c p}$ & $\sigma$ \\
\hline 84,4 & 1,13 & 0,04 & 0,33 & 48,21 & 1,25 & 40 & 106 \\
\hline
\end{tabular}

U/UTP cat 5e $4 \times 2 \times 0,52$ с оболочкой из поливинилхлорида и многопарного кабеля типа U/UTP cat 5 e $25 \times 2 \times 0,52$ с полиэтиленовой оболочкой производства AO "СКК". Электрические характеристики определены с использованием стационарной измерительной системы AESA-9500.

\section{Низкочастотные характеристики}

К низкочастотным параметрам относятся:

- электрическое сопротивление медных жил постоянному току R (норма: не более $95 \mathrm{OM} / \mathrm{KM})$;

- омическая асимметрия жил в рабочей паре $\Delta \mathrm{R}$ (норма: не более $2 \%$ );

- рабочая емкость C (норма: не более 56 нФ/км);

- емкостная асимметрия рабочей пары Е (норма: не более 1600 пФ/км).

Низкочастотные характеристики очень чувствительны к геометрической и структурной неоднородностям медных жил и изоляции. Высокая геометрическая и структурная однородность является основой выполнения норм и стабильности высокочастотных характеристик кабеля.

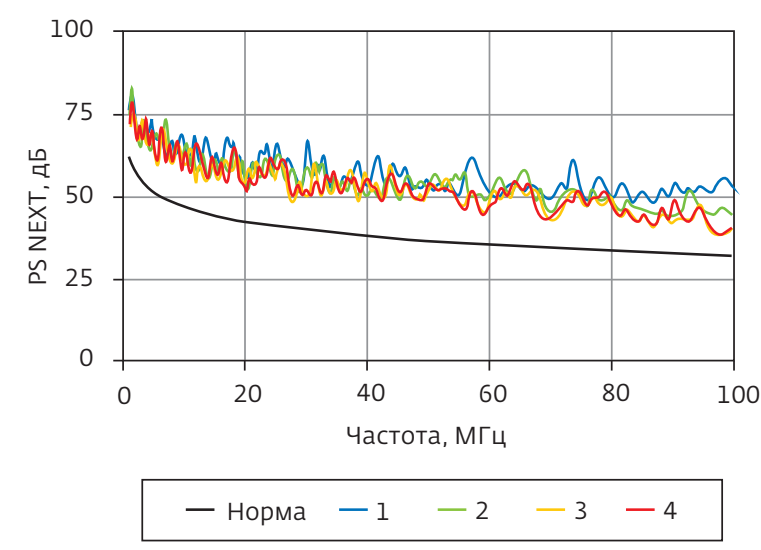

Рис.1. Частотные характеристики суммарного переходного затухания на ближнем конце PS NEXT кабеля U/UTP cat 5e $4 \times 2 \times 0,52$
Для анализа электрических характеристик были взяты результаты 10 протоколов испытаний указанного выше кабеля стандартной длиной 305 м, которые предоставляются потребителю вместе с кабелем. Результаты измерений низкочастотных характеристик отдельных пар кабеля статистически обработаны с учетом того, что они подчиняются нормальному закону распределения и характеризуются статистическим средним значением и среднеквадратическим отклоне нием от среднего значения [4]. Результаты статистической обработки приведены в табл.1. В этой таблице $\sigma$ - среднеквадратическое отклонение параметра.

На основании анализа статистических данных результатов измерения низкочастотных характеристик можно сказать следующее:

- сопротивление медных жил близко к минимальному допуску и очень стабильно;

- омическая асимметрия практически на порядок меньше допустимых 2\%;

- рабочая емкость заметно меньше максимально допустимого значения;

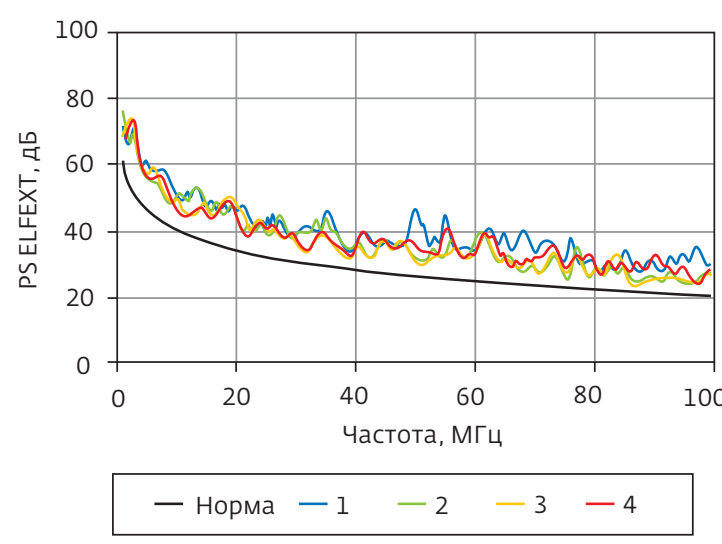

Рис.2. Частотные характеристики суммарной защищенности на дальнем конце PS ELFEXT кабеля U/UTP cat 5 e $4 \times 2 \times 0,52$ 
Таблица 2. Результаты измерения суммарного переходного затухания на ближнем конце PS NEXT (дБ/100 м) кабеля U/UTP cat 5e $4 \times 2 \times 0,52$

\begin{tabular}{|l|c|c|c|c|c|}
\hline \hline $\begin{array}{c}\text { Частота, } \\
\text { МГц }\end{array}$ & Пара 1 & Пара 2 & Пара 3 & Пара 4 & $\begin{array}{c}\text { Нижний } \\
\text { предел }\end{array}$ \\
\hline 1 & 76,76 & 74,98 & 73,43 & 73,95 & 62,30 \\
\hline 4 & 72,42 & 67,05 & 69,28 & 68,74 & 53,30 \\
\hline 10 & 65,05 & 61,71 & 61,66 & 61,65 & 47,30 \\
\hline 16 & 61,05 & 61,92 & 58,84 & 59,46 & 44,30 \\
\hline 20 & 57,25 & 52,40 & 56,99 & 51,49 & 42,80 \\
\hline 31,25 & 56,26 & 59,90 & 59,87 & 54,83 & 39,90 \\
\hline 62,5 & 52,23 & 51,68 & 50,18 & 51,40 & 35,40 \\
\hline 100 & 51,42 & 43,63 & 40,20 & 41,77 & 32,30 \\
\hline
\end{tabular}

- емкостная асимметрия как минимум в пять раз меньше (лучше) допустимой величины.

При этом характеристики четырехпарных и многопарных кабелей практически не отличаются.

\section{Параметры взаимного влияния кабеля типа U/UTP cat 5 e $4 \times 2 \times 0,52$}

В табл.2 и на рис.1 приведены результаты измерения суммарного переходного затухания

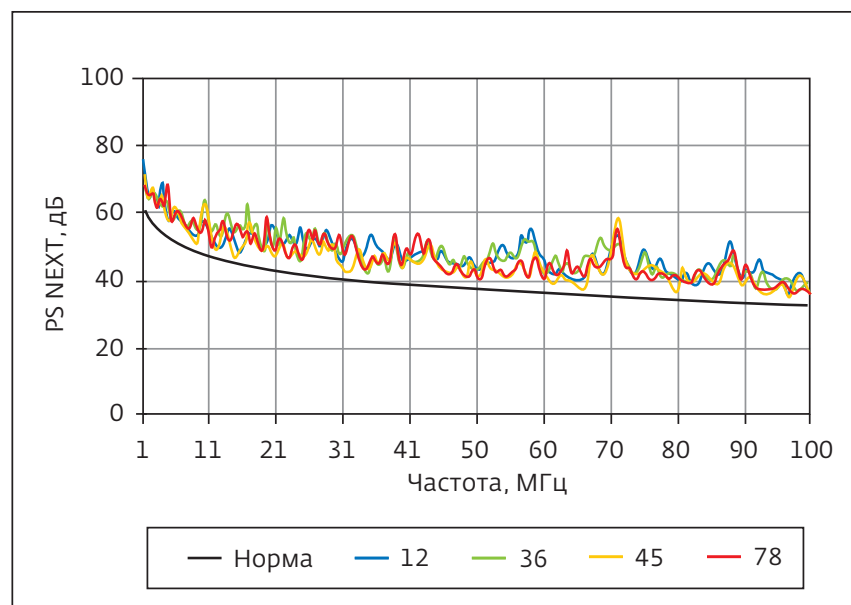

Рис.3. Частотные характеристики суммарного переходного затухания на ближнем конце PS NEXT кабеля U/UTP cat 5e $25 \times 2 \times 0,52$
Таблица 3. Результаты измерения суммарной защищенности на дальнем конце PS ELFEXT (дБ/100 м) кабеля U/UTP cat 5 e $4 \times 2 \times 0,52$

\begin{tabular}{|l|l|l|l|l|c|}
\hline \hline $\begin{array}{c}\text { Частота, } \\
\text { МГц }\end{array}$ & Пара 1 & Пара 2 & Пара 3 & Пара 4 & $\begin{array}{c}\text { Нижний } \\
\text { предел }\end{array}$ \\
\hline 1 & 74,68 & 74,91 & 68,84 & 67,84 & 61,00 \\
\hline 4 & 62,88 & 60,18 & 60,85 & 62,37 & 49,00 \\
\hline 10 & 53,08 & 50,56 & 52,29 & 49,57 & 41,00 \\
\hline 16 & 48,36 & 46,92 & 46,42 & 44,79 & 36,90 \\
\hline 20 & 47,31 & 44,42 & 48,65 & 45,52 & 35,00 \\
\hline 31,25 & 41,04 & 40,62 & 34,23 & 34,07 & 31,10 \\
\hline 62,5 & 40,63 & 35,50 & 35,21 & 38,21 & 25,00 \\
\hline 100 & 31,48 & 26,97 & 27,85 & 26,91 & 21,00 \\
\hline
\end{tabular}

на ближнем конце PS NEXT, а в табл.3 и на рис.2 - суммарной защищенности на дальнем конце PS ELFEXT исследуемого кабеля.

Анализ результатов измерения электрических характеристик взаимных влияний, приведенных в табл.2 и 3, а также на рис.1 и 2 показывает, что исследуемый кабель в диапазоне частот до 100 МГц полностью отвечает установленным нормам ГОСТ Р 54529-2011.

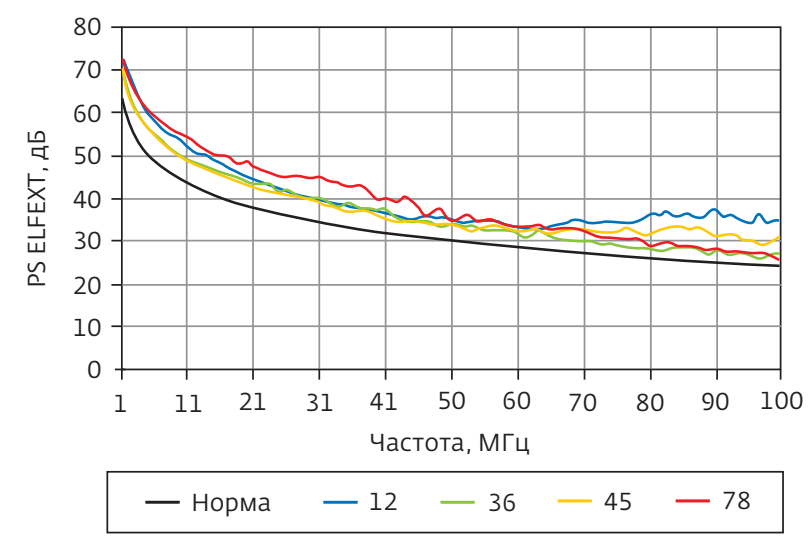

Рис.4. Частотные характеристики суммарной защищенности на дальнем конце PS ELFEXT кабеля U/UTP cat 5e $25 \times 2 \times 0,52$ 
Таблица 4. Результаты измерения суммарного переходного затухания на ближнем конце PS NEXT (дБ/100 м) многопарного кабеля U/UTP cat 5e $25 \times 2 \times 0,52$

\begin{tabular}{|l|c|c|c|c|c|}
\hline \hline $\begin{array}{c}\text { Частота, } \\
\text { МГц }\end{array}$ & Пара 1 & Пара 2 & Пара 3 & Пара 4 & $\begin{array}{c}\text { Нижний } \\
\text { предел }\end{array}$ \\
\hline 1 & 76,2 & 70,8 & 71,3 & 68,9 & 62,3 \\
\hline 4 & 65,2 & 62,2 & 65,0 & 62,0 & 53,3 \\
\hline 10 & 57,2 & 63,2 & 64,6 & 57,9 & 47,3 \\
\hline 16 & 51,0 & 57,9 & 53,1 & 54,4 & 44,3 \\
\hline 20 & 53,7 & 50,4 & 46,9 & 48,9 & 42,8 \\
\hline 31,25 & 41,6 & 52,1 & 41,5 & 50,5 & 39,9 \\
\hline 62,5 & 43,0 & 43,6 & 40,8 & 41,7 & 35,4 \\
\hline 100 & 35,4 & 34,8 & 34,2 & 34,4 & 32,3 \\
\hline
\end{tabular}

\section{Параметры взаимного влияния многопарного кабеля типа U/UTP cat 5e $25 \times 2 \times 0,52$}

Многопарные кабели емкостью $25 \times 2$ изготавливаются на крутильной машине путем скрутки шести четырехпарных пучков в общий сердечник, в центре которого расположена одна пара. Проведенный анализ крутильных машин показал, что для скрутки многопарных LAN-кабелей следует применять машины классической однонаправленной скрутки. Обусловлено это тем, что однонаправленная скрутка по сравнению с разнонаправленной обеспечивает получение геометрически более однородного сердечника, что весьма важно для обеспечения стабильности электрических характеристик. Исследуемый многопарный кабель был изготовлен на крутильной машине клетьевого типа с откруткой.

В табл.4 и на рис.3 приведены результаты измерения суммарного переходного затухания на ближнем конце PS NEXT, а в табл.5 и на рис.4 - суммарной защищенности на дальнем конце PS ELFEXT одного из шести пучков (коричневой расцветки) исследуемого многопарного кабеля емкостью $25 \times 2 \times 0,52$. На рис.3 и 4 характеристики взаимного влияния приведены для пар, образованных проводниками 1 и 2, 3 и 6, 4 и 5, 7 и 8 четырехпарных пучков многопарного кабеля.

Анализ результатов проведенных экспериментальных исследований основных параметров взаимного влияния четырехпарного LANкабеля типа U/UTP cat 5e $4 \times 2 \times 0,52$ и многопар-
Таблица 5. Результаты измерения суммарной защищенности на дальнем конце PS ELFEXT (дБ/100 м) многопарного кабеля U/UTP cat 5e $25 \times 2 \times 0,52$

\begin{tabular}{|l|c|c|c|c|c|}
\hline \hline $\begin{array}{c}\text { Частота, } \\
\text { МГц }\end{array}$ & Пара 1 & Пара 2 & Пара 3 & Пара 4 & $\begin{array}{c}\text { Нижний } \\
\text { предел }\end{array}$ \\
\hline 1 & 73,9 & 69,6 & 69,8 & 72,8 & 61,0 \\
\hline 4 & 61,9 & 58,0 & 57,8 & 62,3 & 49,0 \\
\hline 10 & 53,0 & 50,1 & 49,7 & 54,9 & 41,0 \\
\hline 16 & 47,8 & 46,0 & 45,4 & 50,1 & 36,9 \\
\hline 20 & 44,8 & 43,4 & 42,9 & 48,0 & 35,0 \\
\hline 31,25 & 39,7 & 40,1 & 38,0 & 44,0 & 31,1 \\
\hline 62,5 & 33,9 & 31,4 & 33,0 & 33,6 & 25,0 \\
\hline 100 & 34,5 & 26,7 & 30,6 & 25,3 & 21,0 \\
\hline
\end{tabular}

ного - U/UTP cat 5e $25 \times 2 \times 0,52$ позволяет отметить следующее. В многопарных кабелях нормы на переходное затухание на ближнем и защищенность на дальнем концах хотя и выполняются, но с небольшим запасом. Запас по параметрам взаимного влияния четырехпарных кабелей заметно выше и составляет 5-10 дБ. Это говорит о необходимости уделять особое внимание технологии скрутки сердечников многопарных LAN-кабелей из четырехпарных пучков.

Необходимо минимизировать механические воздействия на кабельный сердечник при его скрутке на крутильной машине. Эти условия наиболее оптимально выполняются при использовании рамочных крутильных машин. Обусловлено это отсутствием в них тяжелых вращающихся масс. Частота вращения относительно легкой малоинерционной крутильной рамки ограничивается лишь ее конструктивной прочностью и может достигать нескольких тысяч оборотов в минуту, что обеспечивает высокую линейную скорость скрутки [6]. В настоящее время такие крутильные машины для однонаправленной скрутки являются основным оборудованием для скрутки сердечников LAN-кабелей.

\section{ЗАКЛЮЧЕНИЕ}

Показано, что высокая геометрическая и структурная однородность изолированных жил, а также применение высокоскоростного специализированного оборудования скрутки пар обеспечивают высокие параметры взаимных влияний четырехпарного LAN-кабеля категории 5e. 
Установлено, что у многопарных кабелей на величину переходного затухание на ближнем и защищенности на дальнем концах оказывает влияние технология скрутки на крутильной машине кабельного сердечника из четырехпарных пучков по причине механических воздействий.

Для минимизации механических воздействий на кабельный сердечник рекомендуется использовать рамочные крутильные машины однонаправленной скрутки с откруткой.

\section{ЛИТЕРАТУРА}

1. ГОСТ Р 54429-2011. Кабели связи симметричные для цифровых систем передачи. Общие технические условия.
2. ISO/IEC 11801. Стандарт телекоммуникационной инфраструктуры коммерческих зданий, 2002.

3. Андреев В., Бульхин А. и др. Качество LANкабелей - основа надежной работы СКС и сетей ШПД // ПЕРВАЯ МИЛЯ. 2020. № 1. С. 24-27.

4. Шварцман В.О. Взаимные влияния в кабелях связи. - М.: Связь, 1996. 368 с.

5. Андреев В.А., Портнов Э.Л., Бурдин В.А. и др. Направляющие системы электросвязи. Учебник для вузов / Под ред. Андреева В. А.; 8-е изд. - М.: Горячая линия-Телеком, 2018. 396 с.

6. Власов Е.В., Парфенов Ю.А. и др. Кабели СКС на сетях электросвязи: теория, конструирование, применение. - М.: Эко-Трендз, 2006. 280 с.

\section{НОВЫЕ КНИГИ ИЗДАТЕЛЬСТВА «ТЕХНОСФЕРА»}

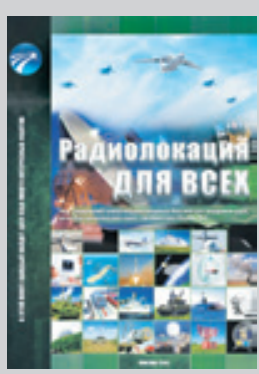

Цена 975 руб.

\section{РАДИОЛОКАЦИЯ ДЛЯ ВСЕХ}

\author{
Верба В. С., Гаврилов К. Ю., Ильчук А. Р., \\ Татарский Б. Г., Филатов А. А. \\ Под ред. Вербы В.С.
}

Настоящая книга одобрена научным советом Российской академии наук «Научные основы построения вычислительных, телекоммуникационных и покационных систем" отделения нанотехнологий и информационных технологий, возглавляемого академиком РАН Ю. В. Гуляевым, как научно-популярное издание

M.: TEXHOCФЕРA, 2020. - 504 C., ISBN 978-5-94836-555-8

Представленный в научно-популярной книге материал можно назвать начальным курсом по радиолокации. В издании рассмотрены основные физические и теоретические вопросы радиолокации, принципы построения радиолокационных систем и основные области их практического использования. Рассмотренные в книге примеры типовых радиолокаторов и области их применений не исчерпывают весь возможный диапазон использования радиолокационной техники и принципов получения информации радиолокационными методами в повседневной жизни человека.

Изложение материала ведется в форме беседы специалиста в области радиолокации - профессора кафедры радиолокации, и студента, мало знакомого с радиотехникой и ее областью радиолокации.

Книга ориентирована в первую очередь на выпускников школ и студентов младших курсов технических вузов, может быть интересна и для студентов старших курсов радиотехнических факультетов, а также для всех интересующихся радиолокацией. 